\title{
Entropy Approach for Volatility of Ethereum and Bitcoin
}

\author{
Ayşe Metin Karakaş \\ Department of Statistic, Faculty of Art and Sciences, \\ Bitlis Eren University, Bitlis, Turkey. \\ Email: aysekarakas5767 [AT] gmail.com
}

\begin{abstract}
The application of entropy in finance can be regarded as the extension of information entropy and probability theory. In this article we apply the concept of entropy for basic crypto money (Ethereum and Bitcoin) to make a comparison. We compute in the first step Shannon entropy with different estimators, Tsallis entropy for different values of its parameter, Rényi entropy and at last the approximate entropy. We provide computational results for these entropies for daily data.
\end{abstract}

Keywords-- Shannon Entropy, Tsallis entropy, Rényi entropy, Approximate entropy, Crypto Money.

\section{INTRODOUCTION}

The history of the word entropy can be traced back to 1865 when the German physicist Rudolf Clausius tried to give a new name to irreversible heat loss, what he previously called equivalent-value. The word entropy was chosen because in Greek, entropies mean content transformative or transformation content [1]. [2] proposed an extension of the concept of entropy, which successfully describes the statistical features of complex systems. [3] determined the cumulative residual entropy, generalized measure of uncertainty which applied in reliability and image alignment and non-additive measures of entropy. [4] suggested a new way of defining entropy of a system, which gives a general form that is non-extensive like Tsallis entropy, but is linearly dependent on component entropies, like Rényi entropy, which is extensive, checked it numerically with the Tsallis and Shannon entropies and indicated constraints on the energy spectra imposed by the properties of the Lambert function, which are absent in the Shannon form. [5] indicated the utility of approximate entropy (ApEn), a modelindependent measure of sequential irregularity, towards this goal, via several distinct applications, both empirical data and model-based, designed cross-ApEn, a related two-variable measure of asynchrony that provides a more robust and ubiquitous measure of bivariate correspondence than does correlation, and the resultant implications to diversification strategies, and supplied analytic expressions for and statistical properties of ApEn, and compare ApEn to nonlinear measures, correlation and spectral analyses, and other entropy measures. [6] indicated that new entropy has the same properties than the Shannon entropy except additive and given that this entropy function satisfies the Lesche and thermodynamic stability criteria. [7] proposed a new method of implementing the principle of maximum entropy to retrieve the risk neutral density of future stock, or any other asset, returns from European call and put prices. [8] defined the market efficiency in foreign exchange (FX) markets by using the multi-scale approximate entropy (MApEn) to assess the randomness in FX market, separated 17 daily FX rates from 1984 to 2011 into there periods by two global events, Southeast Asia currency crisis and American sub-prime crisis and submitted that the developed FX markets is more efficient than emerging FX markets, and that the financial crisis promotes the market efficiency in FX markets significantly, especially in emerging markets, like China, Hong Kong, Korea and African market. In statistical mechanics the interpretation of entropy is the measure of uncertainty about the system that remains after observing its macroscopic properties (pressure, temperature or volume) [9]. [10] considered the most important properties of Rényi divergence and Kullback- Leibler divergence, including convexity, continuity, limits of $\sigma$-algebras, and the relation of the special order 0 to the Gaussian dichotomy and contiguity and indicated how to generalize the Pythagorean inequality to orders different from 1. [11] used to study the complexity of financial time series since the financial market is a complex evolved dynamic system and considered multi scale entropy in the complexity of a time series and applied to the financial market. [12] obtained some integrated techniques for modeling financial data and solving decision making problems, based on risk theory and information theory, examined several risk measures and entropy measures and compared with respect to their analytical properties and effectiveness in solving real problems. [13] described a generalized cumulative residual entropy based on the non-additive Tsallis entropy. [14] considered the effects of financial crises on foreign exchange (FX) markets, where entropy evolution is measured for different exchange rates, using the time-dependent block entropy method and indicated empirical results suggest that financial crises are associated with significant increase of exchange rate entropy, reflecting instability in FX market dynamics. [15] performed such entropy measure on the time series of prices and volatilities of six financial markets on tick-by-tick data sampled every minute for six years of data from 1999 to 2004 and indicated that the entropy of the volatility series depends on the individual market. [16] provided the weighted form of this measure and call it Weighted Cumulative Residual Tsallis Entropy (WCRTE), reproduced ageing classes and shown that it can uniquely determine the survival function and Rayleigh distribution. Bitcoin and Ethereum, generally conducted in the context of 
conceptual explanations, the introduction of cryptocurrency and the volatility of Bitcoin and Ethereum. In this study, we consider the volatility of Bitcoin and Ethereum with entropy method.

\section{MATERİAL AND METHOD}

\subsection{The Shannon Entropy}

The Shannon Entropy of probability measure $p$ on finite set $X$ is given by;

$$
S_{n}(P)=-\sum_{i=1}^{n} p_{i} \ln p_{i}
$$

where $p_{i} \geq 0, i=1,2 \ldots, \mathrm{n}, \sum_{i=1}^{n} p_{i}=1$ and $0 \ln 0=0$. Given a continuous probability distribution with a density function $f(x)$, we can define The Shannon Entropy;

$$
H=-\int_{-\infty}^{+\infty} f(x) \ln f(x) d x
$$

where $\int_{-\infty}^{+\infty} f(x) d x=1$ and $f(x) \geq 0$. The Shannon; in information theory applications, the answer is given by the asymptotic equipartition property;

There is $T \subseteq S^{n} T \subseteq S^{n}$ with,

$$
|T| \leq e^{n(H(\rho)+\varepsilon)}
$$

such that sampling $\mathrm{n}$ times from $\mathrm{p}$ yields an element of $\mathrm{T}$ with probability $\succ 1-\varepsilon$, and $\varepsilon \rightarrow 0$ as $n \rightarrow \infty$ [ $1,2,5,3]$.

\subsection{The Tsallis Entropy}

The Tsallis Entropy, for any positive real number $\alpha$, the Tsallis Entropy of order $\alpha$ of probability measure $p$ on finite set $X$ is defined as [ $1,2,5,3]$

$$
H_{\alpha}(p)=\left\{\begin{array}{cl}
\frac{1}{\alpha-1}\left(1-\sum_{i \in X} p_{i}^{\alpha}\right), & \text { if } \alpha \neq 1 \\
-\sum_{i \in X} p_{i} \ln p_{i}, & \text { if } \alpha=1
\end{array}\right.
$$

The characterization of the Tsallis entropy is the same as that of the Shannon entropy except that for the Tsallis entropy, the degree of homogeneity under convex linearity condition is $\alpha$ instead of 1 .

\subsection{Rényi Entropy}

For $\beta \in[0, \infty]$, the Rényi entropy of order $\beta$ is given by

$$
H_{\beta}(\rho)=\frac{1}{1-\beta} \log \left(\sum_{i \in S} \rho_{i}^{\beta}\right) .
$$

$>$ The scaling factor is conventional: it makes $H_{\beta}$ nonnegative for all $\beta$ and ensures $H_{\beta}\left(u_{n}\right)=\log n$, where $u_{n}$ is the uniform distribution on an $n$ - element set. 
The main property which the Rényi entropies have in common with Shannon entropy is additivity:

$$
H_{\beta}(\rho \times r)=H_{\beta}(\rho)+H_{\beta}(r) \text {. }
$$

Interesting special cases;

$>$ For $\beta=0$, we obtain the max entropy, which is cardinality of the support of $\rho$ :

$$
H_{0}(\rho)=\log |\{i \in S \mid \rho(i) \succ 0\}| \text {. }
$$

For $\beta=1$, we recover Shannon entropy:

$$
\begin{aligned}
H_{1}(\rho) & =\lim _{\beta \rightarrow 1} H_{\beta}(\rho) \\
& =\frac{d}{d \beta}\left(\frac{1}{1-\beta} \log \left(\sum_{i} \rho(i)^{\beta}\right)\right)_{\beta=1}=-\sum_{i} \rho(i) \log \rho(i) .
\end{aligned}
$$

$>$ For $\beta=\infty$, we obtain the min entropy:

$$
H_{\infty}(\rho)=-\log \max _{i} \rho(i)=\log \min _{i} \frac{1}{\rho(i)}
$$

$[1,2,5,3]$.

\section{DATA SET}

We use the daily closing prices of Bitcoin and Ethereum for the period 2015-2018. Table 1 summarizes statistics of Bitcoin and Ethereum data and summarizes statistics of returns series. In Table 2, shows different mean values for the for data set, and the corresponding standard deviations are different. Skewness of returns Bitcoin is negative, indicating that this data is skewed left, Ethereum is skewed right. The high kurtosis of returns reveals that extreme value changes often occur when the tail of return distributions shows fatness. The Jarque-Bera (JB) test shows that the normality of each return series distribution is strongly rejected at 0.05 level, which means all price index distributions are non-normal. Graphical representations of the data employed are shown in figures 1.

Table 1: Summary Statistics

\begin{tabular}{ccc}
\hline & Bitcoin & Ethereum \\
\hline Mean & $6,82 \mathrm{E}+10$ & $2,34 \mathrm{E}+10$ \\
Median & $4,17 \mathrm{E}+10$ & $1,59 \mathrm{E}+10$ \\
Maksimum & $3,27 \mathrm{E}+11$ & $1,35+\mathrm{E} 11$ \\
Minumum & $5,50 \mathrm{E}+09$ & 71176658 \\
Std.Dev & $6,75 \mathrm{E}+10$ & $2,77 \mathrm{e}+10$ \\
Skewness & 1,101806 & 1,349263 \\
Kurtosis & 3,789613 & 4,357657 \\
Jarqure Bera & 247,2577 & 411,7780 \\
Probability & 0,000000 & 0,000000 \\
\hline
\end{tabular}

Table 2: Return Series Summary Statistics

\begin{tabular}{ccc}
\hline & Bitcoin & Ethereum \\
\hline Mean & 0,002116 & 0,004605 \\
Median & 0,002570 & $-0,000301$ \\
Maksimum & 0,225233 & 0,304455 \\
Minumum & $-0,207397$ & $-0,315070$ \\
Std.Dev & 0,040581 & 0,065268 \\
Skewness & $-0,175850$ & 0,252858 \\
Kurtosis & 7,474577 & 6,784658 \\
Jarqure Bera & 908,2276 & 657,2872 \\
Probability & 0,00000 & 0,00000 \\
\hline
\end{tabular}



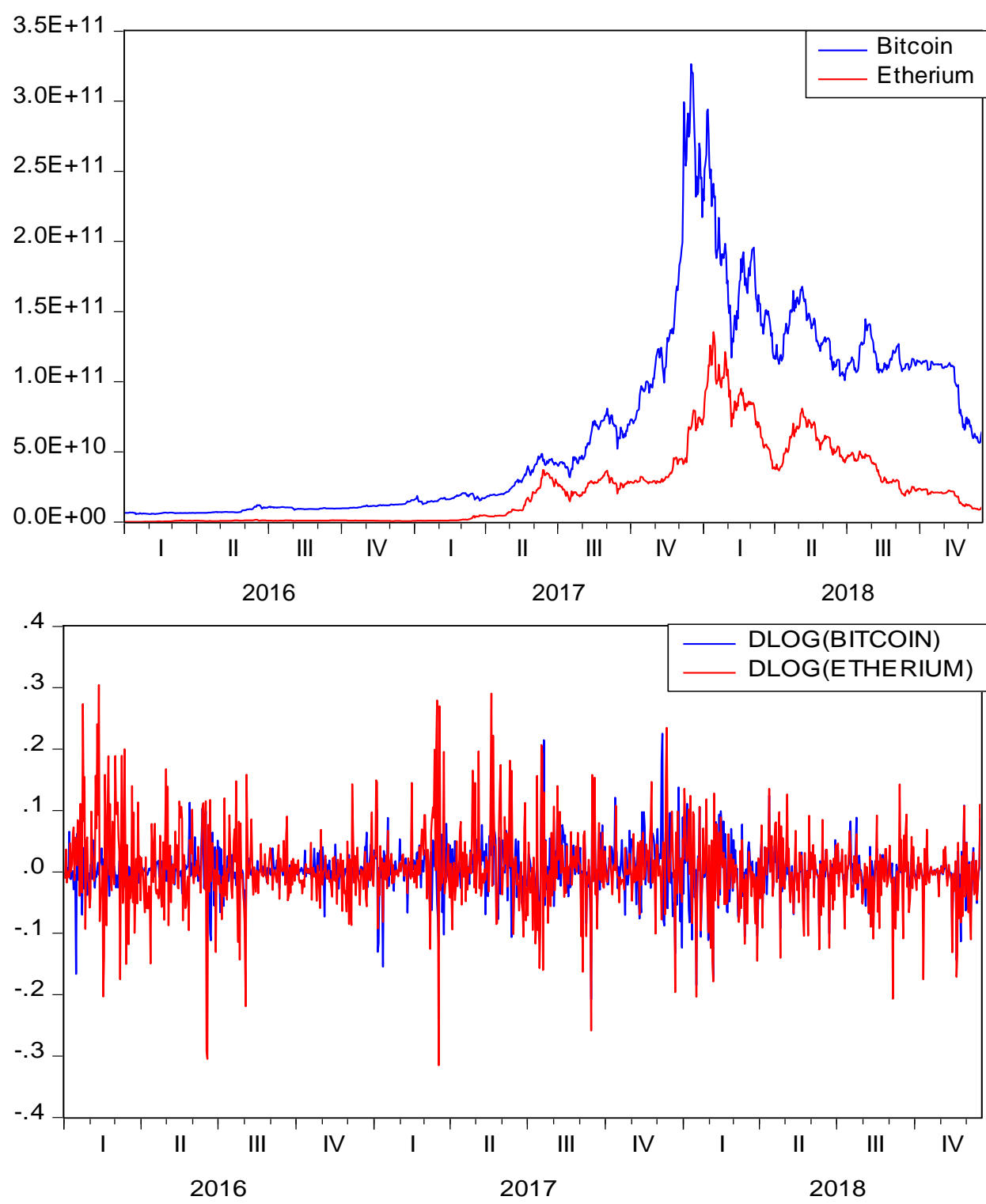

Figure 1: Change over years of BTC and Ethereum series and BTC and Ethereum return series

\section{RESULTS}

We use the entropy method for volatility of Bitcoin and Ethereum. For this, we calculate to Shannon, Tsallis, Rényi and approximate entropies. In Table 3 and Table 4, firstly, we have obtained different estimators (Maximum likelihood (ML), Biased corrected maximum likelihood (MM), Jeffreys (with Dirichlet $\alpha=1 / 2$ ), Laplace (with Dirichlet $\alpha=1 / 2$ ), SG (with Dirichlet $\alpha=1 / 2$ ), CS (Chao Shen Entropy) and Shrink entropy estimators ) for the Shannon entropy measure. Later, we have obtained the Tsallis entropy for different values of $q$ parameter and Rényi entropy measures for different values of $r$ parameter. Finally, we have calculated approximate entropy. When all likely events are same probability, the entropy obtains maximum value. In our empirical results, volatility doesn't show different; this model indicates linear and nonlinear dynamics. For data set, the entropy evaluates the all linear and nonlinear volatility. We obtain form the computational results that all entropies are positive so, characters of our data series are nonlinear. In the daily data series, we obtain that Ethereum series has larger value of approximate entropy. It concludes in this case that Ethereum series is higher than volatility Bitcoin. For the Shannon entropy estimators, it is clear that Ethereum series has larger value, similarly for the Tsallis and Rényi entropy measures, we take attention that $q$ and $r$ approaches to 1, we obtain the Shannon entropy.The conduct of Bitcoin and Ethereum volatility dependes on $q$ and $r$. 
Table 3: Entropy results of Bitcoin Series

\begin{tabular}{lllllll}
\hline \multicolumn{2}{c}{ Shannon } & \multicolumn{2}{c}{ Tsallis } & \multicolumn{2}{c}{ Rényi } & Approximate Entropy \\
\hline \multicolumn{1}{c}{ Method } & Value & $q$ & \multicolumn{1}{c}{ Value } & $r$ & Value & Value \\
\hline ML & 6.317953 & 0 & 1082.0000000 & 0 & 6.987490 & 0.1169102 \\
MM & 6.317953 & 0.2 & 278.6474104 & 0.25 & 6.717101 & \\
Jefferys & 6.317953 & 0.4 & 85.5591539 & 0.5 & 6.530324 & \\
Laplace & 6.317953 & 0.6 & 30.8163806 & 1 & 6.317953 & \\
SG & 6.317953 & 0.8 & 12.9309750 & 2 & 6.113352 & \\
Minimax & 6.317953 & 1 & 6.3179529 & 4 & 5.891448 & \\
CS & 6.317953 & 1.2 & 3.5715128 & 8 & 5.675464 & \\
Shrink & 6.317953 & 1.4 & 2.2922346 & 16 & 5.500457 & \\
& & 1.6 & 1.6257890 & 32 & 5.382175 & \\
& & 1.8 & 1.2408402 & 64 & 5.311846 & \\
& & 2 & 0.9977869 & Infinite & 5.232682 & \\
\hline
\end{tabular}

Table 4: Entropy results of Ethereum Series

\begin{tabular}{lcllllc}
\hline \multicolumn{2}{c}{ Shannon } & \multicolumn{2}{c}{ Tsallis } & \multicolumn{2}{c}{ Rényi } & Approximate Entropy \\
\hline \multicolumn{1}{c}{ Method } & Value & $q$ & \multicolumn{1}{c}{ Value } & $r$ & Value & Value \\
\hline ML & 6.514913 & 0 & 1082.0000000 & 0 & 6.987490 & 0.1390798 \\
MM & 6.514913 & 0.2 & 302.1496142 & 0.25 & 6.836196 & \\
Jefferys & 6.514913 & 0.4 & 94.3142390 & 0.5 & 6.706478 & \\
Laplace & 6.514913 & 0.6 & 33.4013674 & 1 & 6.514913 & \\
SG & 6.514913 & 0.8 & 13.6482137 & 2 & 6.304219 & \\
Minimax & 6.514913 & 1 & 6.5149127 & 4 & 6.089233 & \\
CS & 6.514913 & 1.2 & 3.6261345 & 8 & 5.858163 & \\
Shrink & 6.514913 & 1.4 & 2.3076459 & 16 & 5.681844 & \\
& & 1.6 & 1.6302190 & 32 & 5.567809 & \\
& & 1.8 & 1.2421358 & 64 & 5.499545 & \\
& & 2 & 0.9981714 & Infinite & 5.421030 & \\
\hline
\end{tabular}

\section{CONCLUSION}

In this article we have considered the entropy approach to explain for volatility of Bitcoin and Ethereum. Bitcoin, one of the most popular cryptocurrencies, was introduced in a white paper written by Nakamoto (2008). Ethereum is a rising star in the crypto currency world. It has quickly become the second largest digital currency, booming in value and spurring the rise of hundreds of new rivals to Bitcoin. While Bitcoin is created as an alternative to regular money and is thus a medium of payment transaction and store of value, Ethereum is developed as a platform which facilitates peer-to-peer contracts and applications via its own currency vehicle. In this article, we observed that the volatility of Ethereum is more than bitcoin (figure 1 and table 3,4). Bitcoin and Ethereum as a portfolio item and transaction cost decreasing device.

\section{REFERENCES}

[1] Laidler, K.J., Thermodynamics, In The World of Physical Chemistry, Oxford University Press, New York, NY, USA, pp. 156-240,1995.

[2] Tsallis, C., "Possible generalization of Boltzmann-Gibbs statistics", Journal of Statistical Physics, 52,479-487,1988.

[3] Rao, M.,Chen, Y.,Vemuri, B.C., \& Wang, F., Cumulative residual entropy: a new measure of information , IEEE transactions on Information Theory, 50(6), 1220-1228, 2004.

[4] Shafee, F., "Lambert function and a new non-extensive form of entropy", IMA journal of applied mathematics, 72(6), 785-800, 2007.

[5] Pincus, S., Approximate entropy as an irregularity measure for financial data, Econometric Reviews, 27(4-6), 329$362,2008$.

[6] Ubriaco, M. R., Entropies based on fractional calculus, Physics Letters A, 373(30), 2516-2519, 2009.

[7] Rompolis, L. S., "Retrieving risk neutral densities from European option prices based on the principle of maximum entropy", Journal of Empirical Finance, 17(5), 918-937, 2010.

[8] Wang, G. J., Xie, C., \& Han, F., Multi-scale approximate entropy analysis of foreign exchange markets efficiency, Systems Engineering Procedia, 3, 201-208, 2012. 
[9] Ormos, M., \& Zibriczky, D., Entropy-based financial asset pricing, PloS one, 9(12), e115742, 2014.

[10] Van Erven, T., \& Harremos, P., Rényi divergence and Kullback-Leibler divergence, IEEE Transactions on Information Theory, 60(7), 3797-3820, 2014.

[11] Niu, H., \& Wang, J., Quantifying complexity of financial short-term time series by composite multiscale entropy measure. Communications in Nonlinear Science and Numerical Simulation, 22(1-3), 375-382, 2015.

[12] Dedu, S., \& Toma, A., An Integrated Risk Measure And Information Theory Approach For Modeling Financial Data And Solving Decision Making Problems, Procedia Economics and Finance, 22, 531-537, 2015.

[13] Sati, M. M., \& Gupta, N., "Some characterization results on dynamic cumulative residual Tsallis entropy", Journal of Probability and Statistics, 2015.

[14] Stosic, D., Stosic, D., Ludermir, T., de Oliveira, W., \& Stosic, T., Foreign exchange rate entropy evolution during financial crises. Physica A: Statistical Mechanics and its Applications, 449, 233-239,2016.

[15] Ponta, L., \& Carbone, A., Information measure for financial time series: quantifying short-term market heterogeneity, Physica A: Statistical Mechanics and its Applications, 2018.

[16] Khammar, A. H., \& Jahanshahi, S. M. A., On weighted cumulative residual Tsallis entropy and its dynamic version, Physica A: Statistical Mechanics and its Applications, 491, 678-692, 2018.

[17] Nakamoto, S., Bitcoin: A peer-to-peer electronic cash system, 2008. 